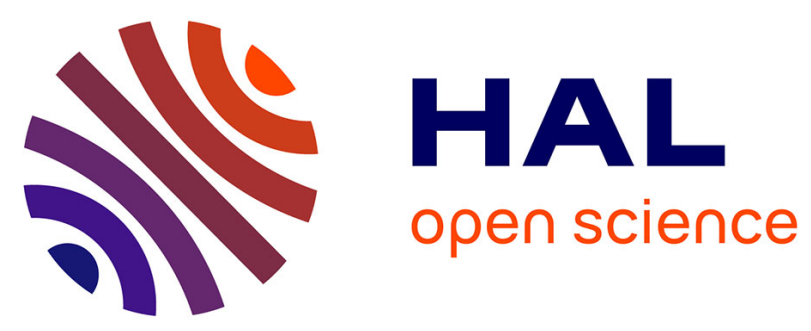

\title{
L'évolution de l'enseignement de l'agronomie à l'INA Paris-Grignon face aux changements sociétaux, scientifiques et institutionnels
}

\author{
Thierry Doré
}

\section{- To cite this version:}

Thierry Doré. L'évolution de l'enseignement de l'agronomie à l'INA Paris-Grignon face aux changements sociétaux, scientifiques et institutionnels. Pour: enquêtes et témoignages, 2009, 200, pp.111118. 10.3917/pour.200.0111 . hal-01361194

HAL Id: hal-01361194

https://hal-agroparistech.archives-ouvertes.fr/hal-01361194

Submitted on 6 Sep 2016

HAL is a multi-disciplinary open access archive for the deposit and dissemination of scientific research documents, whether they are published or not. The documents may come from teaching and research institutions in France or abroad, or from public or private research centers.
L'archive ouverte pluridisciplinaire HAL, est destinée au dépôt et à la diffusion de documents scientifiques de niveau recherche, publiés ou non, émanant des établissements d'enseignement et de recherche français ou étrangers, des laboratoires publics ou privés. 


\section{Thierry DORÉ}

Professeur d'Agronomie à AgroParisTech

\section{L'évolution de l'enseignement de l'Agronomie à l'INA Paris-Grignon face aux changements sociétaux, scientifiques et institutionnels}

L'analyse de l'évolution de l'enseignement de l'Agronomie dans l'enseignement supérieur pendant quatre décennies est un sujet qui demanderait un vrai travail d'historien, ou, à tout le moins, de spécialiste des sciences humaines à même de replacer cette évolution dans un cadre épistémologique, sociologique, politique et économique, le tout en s'appuyant sur une analyse comparée de plusieurs établissements. Je me contenterai pour ma part de déployer un témoignage et non une analyse, et cela sur le seul établissement que je crois suffisamment connaître, à savoir l'Institut national agronomique Paris-Grignon, et sur la période allant de sa création (1971) à sa disparition par fusion dans AgroParisTech (2007) ${ }^{1}$. Sur cette période, la discipline Agronomie et son enseignement ont considérablement évolué. Toutefois, l'Agronomie a gardé une identité et une certaine forme d'autonomie scientifique qui autorisent une analyse diachronique d'un objet clairement identifié : l'étude scientifique des relations entre les plantes cultivées, le milieu (envisagé sous ses aspects physiques, chimiques et biologiques) et les techniques agricoles (définition de l'Agronomie dans les statuts de l'Association française d'Agronomie). Les évolutions de l'enseignement de cette discipline touchent à la fois, et de manière bien entendu interdépendante, les finalités de l'enseignement, l'organisation des cursus, le contenu des enseignements et les méthodes pédagogiques employées. Je distinguerai trois tendances lourdes dans ces évolutions.

\section{La décroissance mais la permanence d'une préoccupation méthodologique et d'une confrontation au terrain}

Dans les années 1970, l'enseignement était très marqué par l'apprentissage de l'exercice de la discipline sur le terrain. Dans le cursus d'ingénieur agronome (le seul représenté à l'époque dans

\footnotetext{
${ }^{1}$ Pour des réflexions plus larges sur l'enseignement de l'agronomie, voir par exemple :

J. Caneill, B. Ney, J. Wery, P. Leterme, "Quel enseignement en agronomie pour les établissements d'enseignement supérieur du ministère de l'Agriculture et de la Pêche ? », Compte-rendu de l'Académie d'agriculture de France $n^{\circ}$ 87(4), p. 277-288, 2001.

T. Doré, «Six points-clés pour repenser l'enseignement en agronomie » in Loi d'orientation agricole, savoirs et qualifications professionnelles ; analyse des enjeux et perspectives pour l'évolution des savoirs, Éducagri éditions, p. 193-195, 2002. P.Leterme, J. Caneill, Adapter la formation en agronomie, Pour n 178, p. 182-191, 2003.
} 
l'établissement), l'Agronomie occupait un volume horaire important en première année. II s'agissait d'y initier les étudiants à la compréhension des fondements de la discipline et au maniement de certains outils. La progression pédagogique était organisée autour du stage en exploitation agricole - alors de longue durée (en trois périodes) et occupant une place centrale dans le cursus de première année qui permettait des allers-retours entre théorie, pratique et observation personnelle. La confrontation à la réalité agricole et agronomique, en groupe lors de séances de travaux pratiques et de travaux dirigés, et de manière individuelle en stage, permettait aux étudiants d'acquérir des connaissances et savoir-faire complémentaires du cours. L'enseignement de deuxième année du même cursus visait à compléter les connaissances et savoir-faire, avec des unités d'enseignement souvent orientées par filière ou système de production. En troisième année, pour les étudiants inscrits dans la spécialisation Sciences et techniques des productions végétales sous la responsabilité du professeur d'Agronomie, on retrouvait de manière beaucoup plus approfondie les mêmes préoccupations. En particulier, il existait une très longue séquence collective (jusqu'à deux mois) sur le terrain, l'étude régionale, qui permettait de réaliser des diagnostics agronomiques approfondis tant sur le fonctionnement des champs cultivés que sur celui des exploitations agricoles. Cette étude, commanditée par des organismes acteurs locaux, contribuait à faire des étudiants d'excellents experts à ces échelles, capables d'intégrer dans leurs analyses et propositions la diversité des milieux et des exploitations ainsi que la compréhension des motivations des acteurs. Par ailleurs, le stage de fin d'étude était réalisé exclusivement dans des laboratoires de recherche, ce qui permettait de compléter l'approche scientifique de l'agriculture et de renforcer la confrontation aux données ce qui, au-delà de l'aspect pédagogique, a permis le développement d'une approche épistémologique originale². Entre 1985 et 1995, cette part de l'agronomie très ancrée au terrain a fondu, du fait notamment des réorganisations pédagogiques globales au sein de l'établissement et d'un rééquilibrage des ambitions de formation en Agronomie entre les trois années du cursus. Ainsi la durée du stage de première année a fortement diminué, entraînant une réorganisation complète de l'enseignement d'Agronomie, dont la durée a par ailleurs considérablement décru au cours de la même période (division de plus de moitié de la durée des cours magistraux). La dimension terrain de l'enseignement, en particulier celle liée à l'étude régionale de troisième année, a également diminué du fait de l'introduction d'autres enseignements (notamment de sciences économiques et sociales). Pour autant, elle n'a jamais totalement disparu. En particulier, la spécialité de troisième année a toujours conservé une confrontation aux réalités à l'échelle parcellaire et de l'exploitation agricole, sous forme de séances valorisant la ferme de Grignon, et de voyages régionaux (certes de durée moindre et aux objectifs pédagogiques plus variés). Par ailleurs, au milieu

\footnotetext{
${ }^{2}$ M. Sebillotte, Préface à L'Agronomie aujourd'hui, T. Doré, M. Le Bail, P. Martin, B. Ney, J. Roger-Estrade (éd.), QUAE
} Éditions, 2006. 
des années 1990, l'introduction dans l'enseignement de deuxième année des séquences (de deux mois) d'Initiation à l'Ingénierie de projet (Inip) a permis de reporter à ce niveau du cursus une partie des objectifs antérieurement portés par la première et la troisième année.

Sur un autre registre, les préoccupations d'ordre méthodologique ont constamment, comme dans beaucoup d'autres disciplines (entre autres les disciplines finalisées), marqué l'enseignement de l'Agronomie. La place manque pour l'illustrer, et j'en mentionnerai uniquement quelques aspects :

- l'affirmation, dans l'enseignement de la discipline, d'objectifs non purement cognitifs, comme « développer chez les étudiants le sens de l'action dans un univers complexe [...], l'aptitude à raisonner en termes probabilistes [...], l'idée que les solutions, mêmes techniques, ont toujours une valeur relative $»^{3}$;

- le maintien, pendant une très longue période, d'un enseignement d'épistémologie dans le cursus de troisième année, et l'introduction, il y a une vingtaine d'années, d'enseignements sur les modes de mobilisation des connaissances agronomiques en situation professionnelle (en s'appuyant notamment sur les interfaces avec des disciplines comme la psychologie et l'ergonomie) ;

- le poids significatif dans le cursus d'enseignements relatifs à la modélisation ou aux méthodes d'acquisition et de traitement de données ;

- la volonté constamment réaffirmée de centrer l'enseignement sur des concepts et méthodes génériques, applicables à une diversité de types de cultures et d'environnement socioéconomiques et pédo-climatiques, plutôt que de décliner dans l'enseignement une agronomie tropicale, une agronomie des régions tempérées, une agronomie des pays développés, etc.

\section{Une ouverture considérable à une diversité de métiers et de domaines d'application}

L'évolution progressive de la structure des premiers emplois occupés par les ingénieurs diplômés de l'école a amené à une transformation progressive des objectifs de formation. À la fin des années 1970, une part encore importante des étudiants issus de la spécialisation de troisième année Sciences et techniques des productions végétales trouvait un emploi dans les secteurs de la recherche et de l'enseignement (35\%), et dans des métiers directement en rapport avec la production végétale ou le conseil (37\%). À la fin des années 1980, la moitié des étudiants issus de la même spécialité se

\footnotetext{
${ }^{3}$ M. Sebillotte, Enseignement de première année, Brochure INA_P_G, 1977.
} 
plaçaient dans l'industrie, le commerce et les services. La co-évolution des enseignements et des métiers s'est traduite par des modifications profondes des enseignements d'Agronomie. Au milieu des années 1980, la spécialisation Sciences et techniques des productions végétales a ainsi considérablement modifié son projet de formation. $Y$ furent introduites des séquences permettant une meilleure préparation aux nouveaux métiers, notamment des enseignements traitant d'interfaces entre la production végétale et l'économie et la gestion, une option de formation sur les filières de production végétale, un accent nouveau mis sur la qualité des produits. Surtout, les stages réalisés par les étudiants se sont largement diversifiés : à la vertu imposée à tous les étudiants de l'apprentissage par la recherche (stage en laboratoire) s'est substituée celle de la confrontation possible à une diversité de métiers (conseil en bureau d'étude, ingénieur qualité/approvisionnement, etc.) - avec un retour très positif vers le corps enseignant, identifiant mieux à partir de ces expériences de stage la diversité des situations professionnelles dans lesquelles l'Agronomie était mobilisée, et les enseignements à construire pour y préparer. En parallèle, l'établissement s'est vu confier au milieu des années 1980 la responsabilité pédagogique d'une formation d'ingénieurs et techniciens du développement agricole (la Relance agronomique), décidée suite au constat d'un besoin de former des agronomes de haut niveau susceptibles, au plan régional, de travailler dans plusieurs types de structures. II s'agissait d'une formation continue assez lourde (sept puis neuf semaines de formation, et un mémoire de trois puis six mois) transformée au début des années 1990 en mastère. Dans une certaine mesure, il y a eu à cette époque transfert à la formation continue d'un certain type d'enseignement de la formation initiale du cursus ingénieur, marqué par la formation d'ingénieurs de terrain proches de la production. Enfin, au milieu des années 1980, la formation de futurs chercheurs s'est autonomisée dans nos établissements, l'Agronomie étant, comme les autres disciplines, concernée par le phénomène. Jusqu'alors, comme on l'a vu, la formation en Agronomie des ingénieurs avait une dimension forte par la recherche, et nombre de ces ingénieurs débutaient leur carrière dans des organismes de recherche, ce qui en faisait également une formation pour la recherche. Dans la deuxième moitié des années 1980, l'établissement a été associé à la responsabilité de Diplômes d'études approfondies (DEA), formant spécifiquement à la recherche; cela permettait d'alléger d'autant, et de manière bienvenue, le cursus de ceux que justement, à ce moment, on cherchait à former à une gamme plus large de métiers. Toutefois, l'agronomie n'étant en France que très peu (et pas du tout en région parisienne) une discipline universitaire, son inscription dans des cursus de DEA n'est pas allée de soi. Des alliances temporaires ont ainsi été passées successivement ou concomitamment avec l'Écologie, la Génétique, la Phytopathologie, la Physiologie végétale, pour des formations de DEA dans lesquelles l'Agronomie 
avait une reconnaissance variable ; ceci jusqu'à la validation en propre en 2002 d'une spécialité de master dédiée à l'Agronomie 4 .

L'enseignement d'Agronomie témoigne également d'une co-évolution avec les secteurs d'emploi des diplômés. On a déjà cité l'accroissement dans les années 1980 de la part de la problématique liée à la qualité des produits et aux filières de production végétale. Cette dimension a continuellement été renouvelée dans les deux dernières décennies, avec l'enrichissement des critères de qualité pris en considération (en particulier critères de qualité sanitaire). Elle a donné lieu à la formation d'enseignements optionnels de troisième cycle très structurés ${ }^{5}$ et à haute valeur ajoutée professionnelle, croisant l'analyse verticale de la filière et l'analyse horizontale de la production à l'échelle d'un territoire et proposant des outils pour comprendre et prévoir l'évolution des cahiers des charges, évaluer les relations des partenaires en présence et proposer des solutions agronomiques limitant ou valorisant la variabilité des résultats de la production végétale. Le déroulement de ces enseignements permettait à l'étudiant de comprendre comment l'agronome mobilise des outils de diagnostic, de modélisation, de prévision à des échelles variées (la plante, la parcelle, la sole et la région) pour répondre à des finalités de production résultant de la confrontation des objectifs de plusieurs acteurs (agriculteurs, industriels, consommateurs, responsables d'aménagement), tout en valorisant les derniers résultats de la recherche en Agronomie sur ces questions, comme le concept de système local d'approvisionnement. Une autre tendance lourde d'ouverture a été celle permettant la prise en charge (facilitée notamment par l'attention accordée antérieurement à la notion de fertilité) des questions d'environnement dès les années 1980, puis d'aménagement du territoire dans les années 1990. Dès 1990, A. Capillon était recruté comme professeur d'agronomie sur une thématique Agriculture/environnement ; lors de sa leçon de concours (intitulée «Utilité et spécificité de l'approche de l'environnement par l'agronome »), il allait insister sur les changements d'objets et d'échelles que requérait cette préoccupation, et développer plusieurs des thématiques largement enseignées à partir de cette époque, liant agriculture et environnement - pollution nitrique des nappes phréatiques, érosion, gestion des effluents d'élevage, rôle des prairies... À cette époque déjà, une option de la spécialisation Sciences et techniques des productions végétales était dédiée aux relations agriculture/environnement.

\footnotetext{
${ }^{4}$ De manière en apparence paradoxale, la formation doctorale a suivi un cheminement inverse. Dans les années 1980 et 1990, les établissements inscrivant des thèses en Agronomie étaient fort peu nombreux, et beaucoup de ces dernières réalisées dans des laboratoires de province étaient inscrites à l'INA_P_G. La réforme des écoles doctorales sur site ayant donné un poids fort aux relations de proximité entre laboratoires et écoles, ces inscriptions ont significativement diminué à partir des années 2000.

5 Voir l'analyse de cette orientation dans M. Le Bail, Programme d'enseignement et de recherche pour le concours de professeur d'Agronomie à l'INA_P_G, 2005.
} 


\section{Un enseignement plus intégré}

L'enseignement d'Agronomie a également évolué en étant de plus en plus intégré dans des séquences d'enseignement pluridisciplinaires. De fait, peut-être pour asseoir - et défendre - la légitimité de la discipline revivifiée dans les années 1960 par S. Hénin, puis M. Sebillotte et J.-P. Deffontaines, peutêtre aussi par héritage d'une organisation de l'enseignement marquée par les chaires disciplinaires, les enseignements de l'Agronomie ont été largement centrés sur la discipline elle-même. Les activités pédagogiques étaient principalement disciplinaires, y compris quand elles concernaient (en première année par exemple) l'exploitation agricole, objet rêvé pour réaliser de la multidisciplinarité. Même si des unités de valeur de deuxième année faisaient déjà se côtoyer des disciplines différentes (autour de la notion de filière, ou de l'aridoculture par exemple), ce n'est vraiment qu'à la fin des années 1980 (lors de la réorganisation de l'enseignement autour du stage de première année), et surtout au début des années 1990, en grande partie pour pouvoir mieux aborder les questions environnementales, que des enseignements réellement pluridisciplinaires se sont mis en place, mêlant Agronomie et sciences du milieu physique. En témoignent notamment la fusion dès 1991/92 des spécialisations Sciences et techniques des productions végétales et Sciences du sol et du bioclimat', ou encore la création d'Inip multidisciplinaires (Agronomie, Chimie analytique, sciences du milieu physique, Droit, Sociologie...) de deuxième année autour de la gestion des bassins versants. En parallèle, outre le renforcement des interfaces avec les sciences économiques et sociales déjà évoqué, on peut noter le développement de montages pluridisciplinaires avec les disciplines concourant à la protection des plantes et la Génétique, d'abord dans la formation à la recherche 7 , puis dans les formations d'ingénieur. Toutefois, ce n'est que depuis les années 2000 que l'Agronomie a commencé à pleinement intégrer ces composantes biotiques dans son appréhension du fonctionnement de l'agro-écosystème, et que les enseignements se sont infléchis pour les prendre en considération.

\section{Les enjeux pour la discipline et son enseignement}

Le bref témoignage ici apporté met en évidence des modifications majeures issues d'un double mouvement. Elles sont tout d'abord le fruit d'une transformation endogène traduisant les changements

\footnotetext{
${ }^{6}$ Cette interface disciplinaire a encore été renforcée avec le recrutement en 2003 d'un professeur sur un profil d'articulation entre Agronomie et Sciences du sol.

7 C'est à cette époque que se produit, notamment lors du contact avec ces disciplines des sciences du végétal, la relative autonomisation disciplinaire, à partir de l'Agronomie, de l'Écophysiologie, illustrée notamment par le recrutement d'un professeur écophysiologiste en 1995.
} 
de la discipline enseignée: élargissement des objets, modification de la manière de considérer l'utilisation de la discipline dans la société dans des activités de développement ou industrielles et forte diversification des interfaces disciplinaires, en constituent des aspects marquants. Ces modifications sont également le reflet d'une adaptation de l'enseignement à des changements importants de contexte, parmi lesquels on peut relever deux forces motrices principales. Tout d'abord des changements sociétaux qui interviennent fondamentalement de deux manières : d'une part à travers la mission assignée à l'établissement dans la formation de ses étudiants en termes de métiers auxquels elle doit préparer (ou re-préparer en formation continue), d'autre part au niveau de la définition des compétences que les futurs diplômés doivent acquérir, avec un formidable élargissement de ces compétences directement lié à l'évolution de la place de l'agriculture dans la société (prise en compte des relations agriculture/environnement notamment). Par ailleurs, l'évolution institutionnelle est bien sûr également source de changements ; j'entends par là à la fois l'évolution du cadrage ministériel (par exemple modification dans l'organisation de la formation à la recherche), et l'organisation interne de l'établissement (réformes successives du cursus de deuxième cycle). Ce bilan (incomplet et subjectif) ouvre beaucoup de questions, que je n'ai pas la place de traiter ici - je me contenterai d'en citer trois (en passant notamment sous silence les interrogations relatives aux évolutions qui n'ont pas eu lieu !). La première porte sur le rôle des femmes et des hommes qui font l'enseignement : les évolutions de l'enseignement en sont-elles si indépendantes, ou au contraire leurs goûts, perceptions, aspirations, compétences sont-ils aussi des facteurs majeurs d'évolution? La seconde a trait aux interactions entre enseignants et étudiants : les évolutions (en termes de caractéristiques sociales, de connaissances à l'arrivée dans l'école, de statut dans la société...) des étudiants et les représentations évolutives qu'a la société de ce qu'est un enseignant du supérieur n'ont-elles pas bouleversé les rapports entre les uns et les autres, affectant aussi l'enseignement ? Enfin, comment et en quoi a pu être assurée la permanence d'une discipline (avec une identité disciplinaire fermement revendiquée ${ }^{8}$ ) quand la manière de l'enseigner bougeait de manière si sensible? Pour conclure, je voudrais juste évoquer quelques-uns des enjeux pour la discipline, et pour son enseignement :

- poursuivre le développement d'une meilleure interaction entre l'Agronomie et d'autres disciplines traitant de la composante biotique du champ cultivé (en particulier l'Écologie) ;

- participer à l'analyse des systèmes alimentaires planétaires ;

- intégrer les connaissances les plus récentes issues de la biologie moderne dans l'Agronomie ;

- mettre en place une Agronomie globale, c'est-à-dire capable d'apporter une contribution relative aux effets des systèmes agricoles sur des problèmes alimentaires ou environnementaux

\footnotetext{
${ }^{8}$ Ainsi qu'en témoigne - peut-être en partie pour conjurer tout risque de dilution ou de désincarnation - la volonté de faire paraître un ouvrage sur la discipline (T. Doré et al. L'agronomie aujourd'hui, QUAE Éditions, 2006).
} 
exprimés à l'échelle planétaire.

Nul doute que les équipes enseignantes, qui ont su faire preuve de souplesse, réactivité et adaptation lors des décennies précédentes, y parviendront !

\section{Remerciements}

L'auteur tient à remercier Jacques Caneill et Marianne Cerf pour leurs précieuses suggestions sur une première version du manuscrit. 